\title{
Endothelial NO synthase and reactive oxygen species mediated effect of simvastatin on vessel structure and function: Pleiotropic and dose-dependent effect on tumor vascular stabilization
}

\author{
YESHAN CHEN $^{1}$, SHENG ZHANG $^{1}$, GANG PENG $^{1}$, JING YU $^{2}$, TAO LIU $^{1}$, \\ RUI MENG $^{1}$, ZHENYU LI ${ }^{1}$, YANXIA ZHAO ${ }^{1}$ and GANG WU ${ }^{1}$ \\ ${ }^{1}$ Cancer Center, Union Hospital, Tongji Medical College, Huazhong University of Science and Technology; \\ ${ }^{2}$ Department of Oncology, The Central Hospital of Wuhan, Wuhan 430022, P.R. China
}

Received November 25, 2012; Accepted January 18, 2013

DOI: 10.3892/ijo.2013.1833

\begin{abstract}
Statins have been confirmed with protective effect to microvessels in diabetic retinopathy, implicated as reducing vascular permeability, maintaining endothelial junction integrity and improving blood perfusion, which are surrogate markers of vascular 'normalization', but no data are currently available on efficacy of statins on tumor endothelial functions. Since statins have been shown to exhibit a biphasic dose-related response on biological behaviour of microvessels, we sought to determine whether statins with bipolar concentrations can ameliorate vessel dysfunction and then increase efficiency of chemotherapeutics in Lewis lung carcinoma and B16F10 melanoma models. Our in vitro study showed that simvastatin reduces hypoxia-induced endothelium leakage in human umbilical vein endothelial cells (HUVEC) in a concentration-dependent manner. In tumor-bearing mice, low-dose simvastatin $(0.2 \mathrm{mg} / \mathrm{kg})$ induced upregulation of endothelial NO synthase (eNOS) skewed vessels to a pericyte-coated and stable pattern, while high-dose simvastatin $(10 \mathrm{mg} / \mathrm{kg})$ remarkably deceased reactive oxygen species (ROS)-induced hypoxia-inducible factor (HIF-1 $\alpha$ ) and vascular endothelial growth factor (VEGF) expression, attenuating VEGF-drived tumor vessel hyperpermeability. These changes ultimately improved intratumoral perfusion and decreased tumor hypoxia. Administration of cisplatin and cyclophosphamide to the simvastatin-treated mice resulted in slower tumor growth. Collectively, simvastatin may promote tumor vessel normal-
\end{abstract}

Correspondence to: Dr Gang Wu and Dr Yanxia Zhao, Cancer Center, Union Hospital, Tongji Medical College, Huazhong University of Science and Technology, Wujiadun Road 156, Wuhan 430022, P.R. China

E-mail: wugangzr@yahoo.com.cn

E-mail: zhaoyanxia2011@yahoo.com

Key words: simvastatin, endothelial NO synthase, reactive oxygen species, vascular endothelial growth factor, vascular normalization ization and show clinical benefit when used in combination with chemotherapeutics.

\section{Introduction}

Pathological angiogenesis and abnormal vessel structures and functions are common features of solid tumors and several non-malignant diseases, such as schwannomas and diabetic retinopathy (DR) $(1,2)$. Vessels in these lesions are highly disorganized and inefficient, and lack pericytes or normal attachment between pericytes and endothelial cells (ECs) (3-5). Such abnormalities of tumor vessels in turn lead to impairment of endothelial barrier function, decreased blood flow and subsequent aggravation of hypoxia in tumor tissues, which result in a poor delivery of anticancer therapeutics and augmentation of radioresistance in tumors. Similar vessel phenotypes are shown in DR characterized by breakdown of the blood-retinal barrier (BRB) that lead to diabetic macular edema and vision loss (6).

By correcting the aberrance in morphological structure and function of vessels, we could normalize the microenvironment of retina in favor of disease control and improve response to other therapies $(7,8)$. The strategies for vascular normalization are likely multifaceted. Substantial evidence indicates that anti-angiogenic treatment could achieve this goal by pharmacological inhibition of vascular endothelial growth factor (VEGF) signaling and it has become a widely accepted treatment for several diseases where neovascularization and permeability plays a pivotal role, including cancer and retinal disorders $(8,9)$. Bevacizumab, a humanized, monoclonal anti-VEGF antibody has been applied clinically for this reason. Nonetheless, other strategies exist with distinct efficacy.

3-Hydroxy-3-methylglutaryl-CoA (HMG-CoA) reductase inhibitors, or statins, that have been reported to exert impressive beneficial effects, such as anti-inflammation, antioxidation, protecting against cardiovascular events and DR and anticancer efficacy via lipid-independent mechanisms (10-12). Statins have been shown to maintain endothelial tight junc- 
tions and markedly reduce retinal microvascular permeability through mitigation of oxidative stress and inflammatory status by downregulating reactive oxygen species (ROS) generation, VEGF and intercellular adhesion molecule (ICAM-1) expression in diabetic animals $(10,11,13)$. Oxidative stress and elevated ROS generation have been incriminated in many pathological conditions, including diabetes and cancer $(14,15)$. Hypoxia and other adverse stimuli inducing high levels of ROS contribute to endothelium dysfunction and apoptosis in both diabetic retina and tumor microenvironment (16-18). Additionally, ROS were found to increase hypoxia inducible factor (HIF)-1 $\alpha$ and VEGF expression in cancer cells, suggesting a role of ROS in regulation of angiogenesis and tumor growth (19). Statins, act as an anti-oxidant, scavenging redundant oxygen radicals and hence modulate endothelial barrier functions. Indeed, biphasic effects of statins on angiogenesis have been observed in microvascular endothelium. Low-dose statins promote proliferation and migration of ECs via stimulation of the PI3K-Akt kinase pathway, resulting in the activation of endothelial NO synthase (eNOS) and concomitantly increasing endothelial nitric oxide (NO) production $(20,21)$. Despite the generally acknowledged impression of $\mathrm{NO}$ as a proangiogenic factor, eNOS-derived NO has been shown to induce pericyte recruitment and coverage to immature angiogenic vessels and subsequent stabilization of angiogenic vessels in tumor models (22), which are representative hallmarks of vascular normalization. On the other hand, high concentration of statins exert anti-angiogenic activity by markedly depleting ROS production and inhibiting synthesis of VEGF and activation of HIF-1 $\alpha$, key mediators that involve in amelioration of vascular hyperpermeability $(11,20)$.

Along the pleiotropic actions of statins on microvascular endothelium mentioned above, in the present study, we have tested the hypothesis that simvastatin, a second generation of statins, is a new therapeutic agent that may induce tumor vascular normalization when given at both low and high doses in syngeneic C57BL/6 tumor-bearing mice, however, mediated by probably different underlying mechanisms, as low-dose simvastatin stress on facilitating mural cell recruitment and vessel stabilization and high-dose simvastatin mainly acts on attenuating ROS-eliciting vascular leakage, which was validated by administration of another strong ROS scavenger, diphenyleneiodonium chloride, in the tumor models.

\section{Materials and methods}

Cells and regents. B16F10 melanoma and Lewis lung carcinoma (LLC) cells (ATCC, Rockville, MD) were cultured in RPMI-1640 supplemented with 10\% FBS (Gibco, Grand Island, NY). Human umbilical vein endothelial cells (HUVEC, ATCC) were maintained in endothelial cell medium (ECM, ScienCell, San Diego, CA) adding EC growth supplement and $5 \%$ FBS. For in vitro experiments, simvastatin prodrug (Sigma, St. Louis, MO) was activated to its active forms as described (23). FITC-labelled lectin (Lycopersicon esculentum) was purchased from Vector Laboratories (Lowelville, $\mathrm{OH}$ ). Diphenyleneiodonium chloride (DPI) were purchased from Calbiochem (San Diego, CA). 2',7'-dichlorodihydrofluorescein diacetate (CM-H ${ }_{2}$ DCFDA) were obtained from Molecular Probes (Invitrogen, Carlsbad, CA).
Measurement of EC permeability. To determine the permeability of HUVEC monolayer using FITC-albumin penetrate assays, HUVEC were seeded onto gelatin-coated polystyrene filter inserts (Costar Transwell, no. 3470, 6.5-mm diameter, $0.4-\mu \mathrm{m}$ pore size) at a density of $3 \times 10^{5}$ cells/insert in a final volume of $250 \mu \mathrm{l} \mathrm{ECM}$ with supplements. The cells were grown to high confluence at $37^{\circ} \mathrm{C}$. Subsequently, cells were treated with indicated concentrations $(0.1,2$ and $5 \mu \mathrm{M})$ of simvaststin for $24 \mathrm{~h}$ in the culture medium under either standard cell culture conditions $\left(21 \% \mathrm{O}_{2}\right.$, normoxia) or hypoxia conditions $\left(3 \% \mathrm{O}_{2}\right)$. After which the inserts were transferred into a new 24-well plate containing serum-free media. FITClabeled albumin (Sigma) suspended in serum-free media was added to the EC monolayers to a final concentration of $100 \mu \mathrm{M}$. The transit extent of FITC-albumin across the monolayer was assessed by measuring the rise of FITC-albumin in the lower chamber after $60 \mathrm{~min}$ and fluorescence density was quantified using a spectrofluorometer at an excitation wavelength of $485 \mathrm{~nm}$ and an emission wavelength of $535 \mathrm{~nm}$. The data are reported as relative permeability in which the control group under normoxia condition was set to one.

Animal tumor models. Murine LLC $\left(2 \times 10^{6}\right)$ and B16F10 melanoma cells $\left(2 \times 10^{5}\right)$ were injected s.c. into the right flank of syngeneic female C57BL/6 mice (HFK Bioscience Co., Beijing, China) at 6-8 weeks of age. Tumor volumes $\left(\mathrm{mm}^{3}\right)$ were measured with a caliper (length $\mathrm{x}$ width ${ }^{2} \mathrm{x} \pi / 6$ ). Treatment were initiated when tumors reached a size of $\sim 100 \mathrm{~mm}^{3}$ (6 days for B16 and 8 days for LLC). Animals were randomly selected to receive daily gastric gavage of simvastatin dissolved in $0.25 \%$ carboxymethylcellulose (CMC)/PBS solution at 0.2 and $10 \mathrm{mg} / \mathrm{kg}$ body weight or same amount of vehicles for 7 days. For all animal experiments, tumor-bearing mice were anesthetized using $2 \%$ pentobarbital sodium and the experimental protocols were approved by the Animal Care Committee of Huazhong University of Science and Technology (SYXK 2010-0057).

Tumor perfusion and vessel permeability. Perfused tumor vessels were visualized by tail intravenous injection of $0.05 \mathrm{mg}$ FITC-labeled lectin $(1 \mathrm{mg} / \mathrm{ml}$ in $0.9 \% \mathrm{NaCl})$ in tumor-bearing mice. Fluorophores were allowed to circulate for $10 \mathrm{~min}$ in mice before intracardiac perfusion and fixation by injection of saline (5 $\mathrm{min})$ and $2 \%$ paraformaldehyde $(7 \mathrm{~min})$. Tumors were then harvested and immediately frozen in optimum cutting temperature (OCT) compound (Sakura) in the dark. Thick $(40 \mu \mathrm{m})$ tumor sections were incubated with rabbit anti-CD31 antibody (1:50; Abcam) at $4^{\circ} \mathrm{C}$ overnight and subsequently with DyLight 594-conjugated secondary antibody (1:200; Jackson ImmunoResearch). Vascular leakage was assessed by i.v. injection of $100 \mu \mathrm{l}$ of $2 \%$ Evans blue dye (Sigma) and allowed to circulate for $20 \mathrm{~min}$ before mice were perfused, the tumors were then excised and the Evans blue dye was extracted from the tumor by incubation with $1 \mathrm{ml}$ of formamide at $55^{\circ} \mathrm{C}$ for $16 \mathrm{~h}$. Concentration of the dye was quantified by spectrophotometer at $630 \mathrm{~nm}$.

Contrast-enhanced ultrasonography. Tumor blood flow was assessed using a color Doppler flow imaging system with a broadband 1-5 MHz probe (iU22 SonoCT, Philips, The 
Netherlands). Tumor-bearing mice were placed on a $37^{\circ} \mathrm{C}$ electric warming plate anesthetized and i.v. injected with $0.1 \mathrm{ml}$ of microbubble contrast agent (SonoVue, Bracco, Italy) diluted in $5 \mathrm{ml}$ of $0.9 \%$ saline and images were recorded starting immediately before the injection and continuing $30 \mathrm{sec}$ at a frame rate of $0.5 \mathrm{~Hz}$ to minimize microbubble destruction. A region of interest that contained abundant blood flow signals in transverse plane of tumor was drawn and analyzed by QLAB quantitative technique, vascularity index (VI) and vascularization flow index (VFI) reflecting the amount and intensity of blood flow signal were calculated.

Immunohistochemistry and immunofluorescence. Hypoxia assessment was performed by immunohistochemical staining for HIF-1 $\alpha$ in formalin-fixed, paraffin-embedded $4-\mu \mathrm{m}$ thick tumor serial sections using a rabbit anti-HIF-1 $\alpha$ antibody (1:50, Bioss, Beijing, China). Peroxidase-conjugated goat antirabbit IgG antibodies and avidin-biotin complex-histostain kit (Zhongshan Goldenbridge, Beijing, China) were used for revelation; sections were finally counterstained with Mayer's hematoxylin. For necrosis assay, LLC and B16 tumor sections were stained with hematoxylin and eosin and evaluated by pathologist to confirm necrotic area in tumor tissues. Immunofluorescent staining of pericyte around tumor vessels was detected in frozen tumor sections that were prepared as mentioned above. Frozen tissue sections were co-immunostained with rabbit anti-CD31 (1:50) and Cy3-conjugated mouse anti- $\alpha$-smooth muscle actin ( $\alpha$-SMA) (1:400; Sigma) antibodies, followed by a DyLight 488-conjugated goat antirabbit secondary antibody (1:200; Jackson ImmunoResearch). Images were captured using a confocal microscope (LMS510, Zeiss, Germary). The pericyte coverage index was estimated and presented as the percentage of blood vessels stained for CD31 with $\alpha$-SMA positive pericytes. For all immunostaining assays, five locations from each tumor were randomly sampled and 6-8 tumors per group were analyzed. All images were analyzed by Image-Pro Plus 6.0 software (Media Cybernetics, MD).

Western blot analysis. To detect eNOS, VEGF and HIF-1 $\alpha$ expression level in tumor tissues, equal amounts of protein extracts $(50 \mu \mathrm{g})$ were loaded on $12 \%$ SDS-PAGE and transferred onto a nitrocellulose membrane (Millipore, Billerica, MA). After blocking with 5\% casein/TBST, the membrane was incubated with rabbit anti-eNOS (1:100; Boster, Wuhan, China), rabbit anti-HIF-1 $\alpha$ (1:50), rabbit anti-VEGF (1:200; Santa Cruz, CA) or mouse anti- $\beta$-actin $(1: 1,000$; Santa Cruz, CA) antibody as an internal control. Protein blots were visualized using Super Signal Chemiluminescent kit (Pierce, Rockford, IL). The chemiluminescent signal on X-ray film was scanned and analyzed by Quantity One software (BioRad, CA).

In situ ROS detection and DPI treatment. Intratumoral ROS production was measured in situ on tumor slices as previously described using a fluorescent ROS probe, $\mathrm{H}_{2}$ DCFDA, a substrate without fluorescence itself, converts to a green fluorescent product when it is hydrolyzed by intracellular esterases (24). Briefly, tumor tissues of interest were freshly dissected in $1 \mathrm{X}$ PBS and then 60-80 $\mu \mathrm{m}$ thick frozen tumor sections were prepared for incubation with the freshly prepared $\mathrm{H}_{2}$ DCFDA $(10 \mu \mathrm{M})$ for $15 \mathrm{~min}$ in dark at room temperature. After three washes with PBS, sections were immediately monitored by a confocal microscope and the fluorescent intensity of DCF was semiquantified using Image-Pro Plus 6.0 software (13). To determined alterations after ROS clearance, tumor-bearing mice received an intraperitoneal injection of DPI (a NADPH oxidase inhibitor) or same amount of vehicle for 5 days.

Synergy determination. Subcutaneously implanted LLC and B16 tumors were established as described above. Tumors were allowed to grow to $\sim 100 \mathrm{~mm}^{3}$ (LLC, day 8; B16, day 6) and then tumor-bearing mice were randomly divided into four groups (6-8 mice each group). Group 1 served as control; group 2 received i.p. injection of chemotherapeutics (cisplatin for LLC tumors at a dose of $1.25 \mathrm{mg} / \mathrm{kg}$ on days 9, 11 and 13; cyclophosphamide (CTX) for B16 tumors at a dose of $100 \mathrm{mg} /$ $\mathrm{kg}$ on days 7 and 11 and vehicle $(0.25 \% \mathrm{CMC}, 0.2 \mathrm{ml})$ by gavage; group 3 received cisplatin or CTX (dose regimen as stated above) $24 \mathrm{~h}$ after $0.2 \mathrm{mg} / \mathrm{kg}$ simvastatin (dissolved in $0.25 \% \mathrm{CMC}$, by gavage for 7 consecutive days) treatment; group 4 were given the same chemotherapeutic modality $24 \mathrm{~h}$ after $10 \mathrm{mg} / \mathrm{kg}$ simvastatin daily treatment. Tumor volume was determined every other day till the end of treatment (day 15 for LLC and day 13 for B16) by measurement with calipers.

Statistical analysis. The data are expressed as mean \pm SEM. Differences among groups were evaluated by ANOVA and the unpaired Student's t-test using SPSS 17.0 software. Statistical significance was set at $\mathrm{p}<0.05$.

\section{Results}

Simvastatin alleviates hypoxia-induced endothelium permeability in HUVEC in a concentration-dependent manner. The transfer activity of a labelling high-molecular protein (FITC-albumin, MW, 66,000) across the EC monolayers was measured to evaluate the efficacy of simvastatin on endothelial barrier function in vitro. Permeability increased significantly in HUVEC monolayers that were exposed to hypoxia for $24 \mathrm{~h}$. Various concentrations of simvastatin had no effect on normoxia cells, but markedly decreased permeability in hypoxia cells. The reduction was concentration-dependent. Hypoxia-induced albumin passage was slightly decreased with low concentration $(0.1 \mu \mathrm{mol} / \mathrm{l})$ of simvastatin but was reduced significantly in $2 \mu \mathrm{mol} / 1$ simvastatin (albumin transmit $\mathrm{p}<0.05$ vs. hypoxia control cells) and the decline was maximal at $5 \mu \mathrm{mol} / 1$ simvastatin ( $\mathrm{p}<0.05$ vs. hypoxia control cells) (Fig. 1). Higher concentrations were not available due to their effect on basal EC barrier permeability.

Biphasic doses of simvastatin improve perfusion and inhibit leakiness of tumor vessels. To investigate whether the tumor vessels treated with simvastatin (low- and high- doses) were more efficient, we studied perfusion of tumor vasculature by delivery of FITC-lectin and measuring the proportion of vessels stained for CD31 (all vessels, red) that were co-immunostained with FITC-lectin (functional vessels, green) in the bloodstream using confocal microscopy in mice bearing LLC tumor and B16 melanoma. Staining for EC marker CD31 showed that vessels 


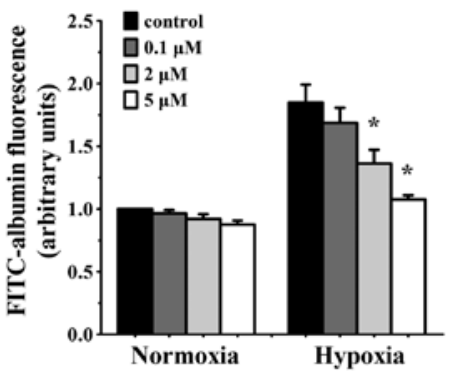

Figure 1. Simvastatin deceases hypoxia-induced endothelium permeability in HUVEC in a dose-dependent manner. FITC-albumin transit across endothelial monolayer was significantly reduced in response to $2 \mu \mathrm{M}$ simvastatin under hypoxia condition and the reduction was maximal at $5 \mu \mathrm{M}$. Simvastatin was unable to decrease EC permeability in HUVEC under normoxia condition. Results shown are representative of three independent experiments and expressed as relative value of normoxia control cells. ${ }^{*} \mathrm{p}<0.05$ versus hypoxia control cells. from both untreated tumors had chaotic structural patterns with increased tortuosity and dilatate lumina and only very few vessels had lectin staining $(12.42 \pm 1.66 \%$ for LLC tumors and $14.23 \pm 0.91 \%$ for B16 tumors, Fig. $2 \mathrm{~A})$. In contrast, low-dose $(0.2 \mathrm{mg} / \mathrm{kg} / \mathrm{d})$ simvastatin significantly increased the amount of lectin stained vessels $(47.62 \pm 3.11 \%$ for LLC tumors and $41.05 \pm 4.05 \%$ for B16 tumors, $\mathrm{p}<0.05$ vs. controls, Fig. 2A-C) although overall vessel density was increased simultaneously to some extent (Fig. 2D and E). Moreover, after high-dose simvastatin $(10 \mathrm{mg} / \mathrm{kg} / \mathrm{d})$ treatment, tumor vessels appeared more sharply and discretely outlined (Fig. 2A) and the vascularity was markedly reduced accompanied by a great majority of vessels perfused as the proportions of lectin-stained vessels in LLC $(83.15 \pm 3.57 \%, \mathrm{p}<0.05)$ and B16 tumors $(73.55 \pm 5.32 \%$, $\mathrm{p}<0.05)$ were greater than that in control animals (Fig. 2B-E).
$\mathbf{A}$

LLC

B16
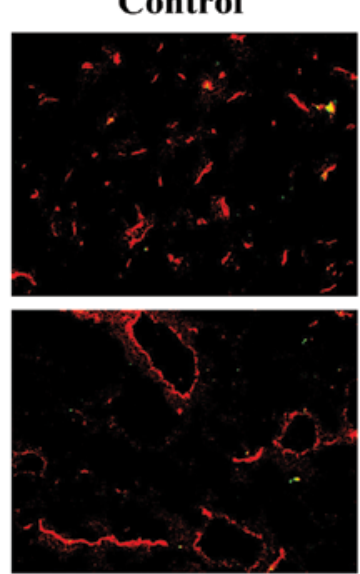

CD31/Lectin
$0.2 \mathrm{mg} / \mathrm{kg} \mathrm{Sim}$
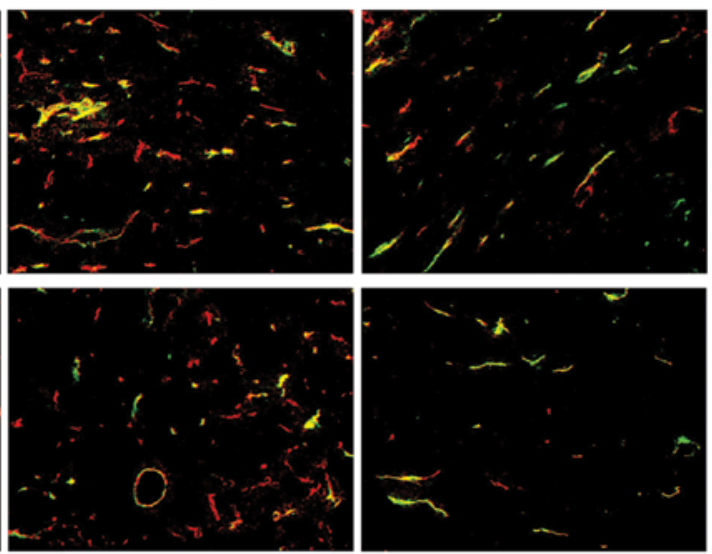

\section{$10 \mathrm{mg} / \mathrm{kg} \mathrm{Sim}$}

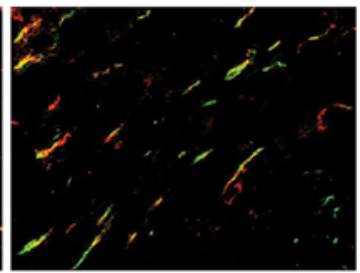

B
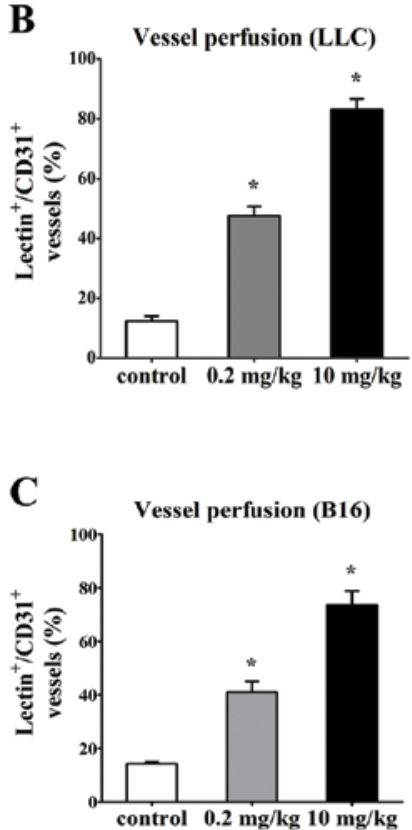

D

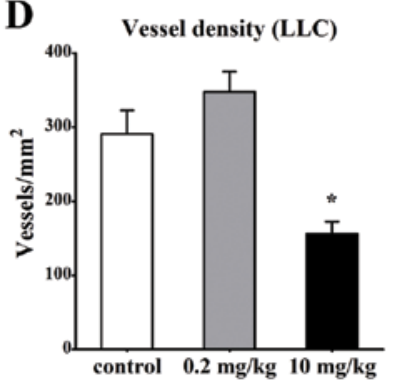

$\mathbf{F}$

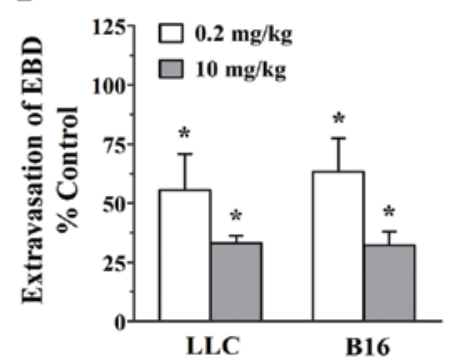

$\mathbf{E}$

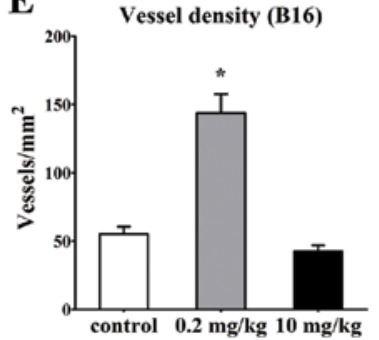

Figure 2. Simvastatin improves tumor vessel perfusion and alleviates vessel leakage. (A) Double staining for CD31 (red) and FITC-conjugated lectin (green) in control, 0.2 and $10 \mathrm{mg} / \mathrm{kg}$ simvastatin-treated LLC and B16 tumors, showing more perfused lectin ${ }^{+} \mathrm{CD} 31^{+}$vessels (\% of CD31 ${ }^{+}$vessels) in 0.2 and $10 \mathrm{mg} / \mathrm{kg}$ simvastatin treated LLC (B) and B16 (C) tumors (n=6). (D and E) Quantitation of tumor vessel density of LLC (D) and B16 (E) tumors. (F) Evans blue dye assay shows significantly decreased vessel leakiness in LLC and B16 tumors treated by 0.2 and $10 \mathrm{mg} / \mathrm{kg}$ simvastatin for 7 days ( $\mathrm{n}=5$ ). Fluorescent images of the sections were captured at magnification $\times 200 .{ }^{*} \mathrm{p}<0.05$ versus corresponding control animals. 
A
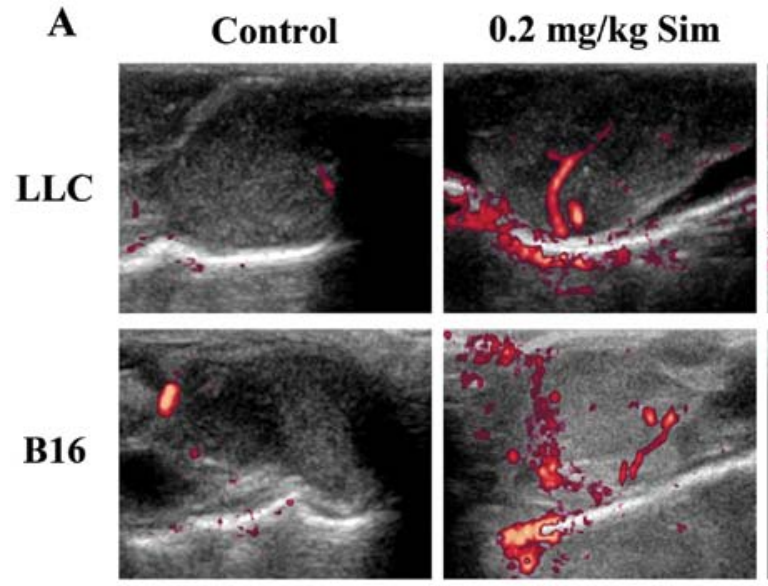

$10 \mathrm{mg} / \mathrm{kg} \mathrm{Sim}$

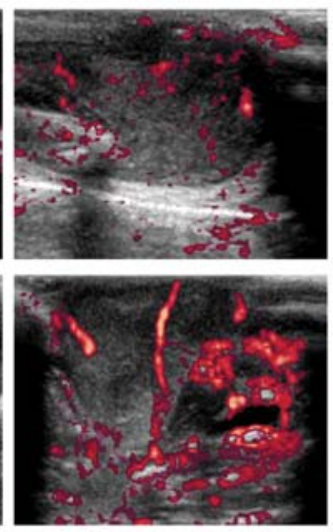

B

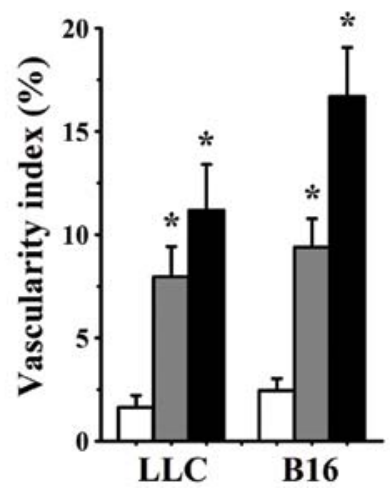

C

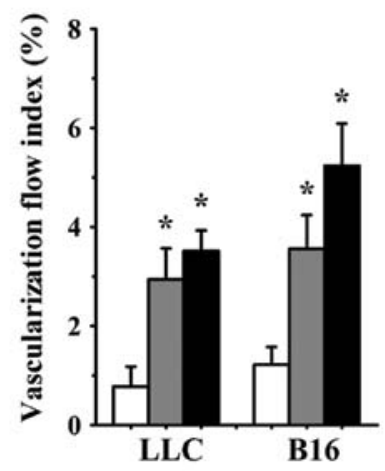

Figure 3. Simvastatin increases tumor blood flow in vivo. (A) Color Doppler flow imaging shows enhanced blood flow signal (red) in 0.2 and 10 mg/kg simvastatin treated subcutaneous LLC and B16 tumors compared with controls. Quantification of vascularity index (B) and vascularization flow index (C) using QLAB quantitative technique, reflecting amount and intensity of blood flow signal in tumors $(\mathrm{n}=6)$. * $\mathrm{p}<0.05$ versus corresponding control animals.

These data implicate an effective role of simvastatin at increasing functional blood vessels in tumor.

Next, the vessel permeability in tumor models was directly assessed by comparing the extravasation of Evans blue dye into the interstitium of treated tumors, with control ones and we found that simvastatin treatment significantly decreased vessel leakiness in LLC and B16 tumors. After a 7-day treatment, Evans blue dye extravasation into low- and high-dose treated LLC tumors were reduced to $55.47 \pm 15.36$ and $33.03 \pm 3.19 \%$ $(\mathrm{p}<0.05)$, the degree of Evans blue dye extravasation in sizematched controls; corresponding data regarding Evans blue dye extravasate reduction in biphasic-dose treated B16 tumors were $63.33 \pm 14.04 \%(\mathrm{p}<0.05)$ and $32.17 \pm 5.68 \%(\mathrm{p}<0.05)$, respectively (Fig. 2F).

Simvastatin treatment increases tumor blood flow. In order to further determine whether alterations of tumor vessel structure and function could affect tumor blood flow after simvastatin treatment, we used contrast-enhanced Doppler imaging to evaluate signal amount and intensity of blood flow in tumor models. Simvastatin treatment increased vascularity index (VI) and vascularization flow index (VFI) in LLC tumors at 0.2 and $10 \mathrm{mg} / \mathrm{kg} / \mathrm{d}$ on day 7 ( $\mathrm{p}<0.05$ vs. control for both), likewise, analysis of VI and VFI confirmed a significant increase of blood flow in 0.2 ( $\mathrm{p}<0.05$ vs. controls) and $10 \mathrm{mg} / \mathrm{kg} / \mathrm{d}$ ( $<<0.05$ vs. controls) simvastatin-treated B16 tumors (Fig. 3).
Effect of simvastatin on tissues hypoxia and necrosis. Considering that correction of vessel structural abnormalities improves blood flow in simvastatin-treated mice, we considered that simvastatin, by ameliorating tumor blood flow, would hence reduce hypoxia and necrosis in tumor tissues. HIF-1 $\alpha$ staining by histochemistry assays in tumor sections was used to assess the degree of hypoxia in tumor microenvironment. As we expected, number of HIF-1 $\alpha$-positive cells were reduced in LLC and B16 tumors in response to $0.2 \mathrm{mg} / \mathrm{kg} / \mathrm{d}$ simvastatin as compared with untreated controls $(115 \pm 9 \mathrm{HIF}-1 \alpha /$ field in LLC tumor vs. $150 \pm 10 \mathrm{HIF}-1 \alpha /$ field in control animals and $133 \pm 8$ $\mathrm{HIF}-1 \alpha /$ field in B16 tumors vs. $171 \pm 12 \mathrm{HIF}-1 \alpha /$ field in control ones, p<0.05, Fig. 4A and B). Also, $10 \mathrm{mg} / \mathrm{kg} / \mathrm{d}$ simvastatin groups showed lesser HIF-1 $\alpha$-positive cells in LLC (62 \pm 5 $\mathrm{HIF}-1 \alpha /$ field $)$ and B16 (76 $\pm 5 \mathrm{HIF}-1 \alpha /$ field) tumors than control animals ( $\mathrm{p}<0.05$ for both, Fig. 4A and B). Our western blotting data further confirmed that HIF-1 $\alpha$ expression was decreased significantly in both LLC and B16 tumors treated with 0.2 and $10 \mathrm{mg} / \mathrm{kg} / \mathrm{d}$ simvastatin (Fig. 4C).

As assessed by H\&E staining at day $7,0.2 \mathrm{mg} / \mathrm{kg}$ simvastatin-treated tumors displayed smaller necrotic area $(9 \pm 2$ vs. $44 \pm 4 \%$ for control LLC tumors and $12 \pm 4$ vs. $53 \pm 7 \%$ for control B16 tumors, p<0.05, Fig. 4D and E). Also, the necrotic tumor area was significantly decreased in $10 \mathrm{mg} / \mathrm{kg}$ simvastatin treated LLC $(7 \pm 2 \%, \mathrm{p}<0.05)$ and B16 tumors $(8 \pm 2 \%, \mathrm{p}<0.05$, Fig. 4D and E). 

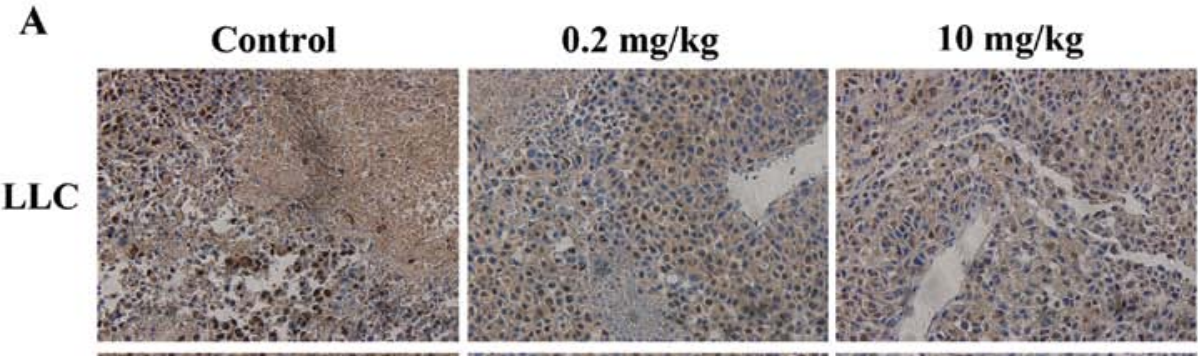

B16
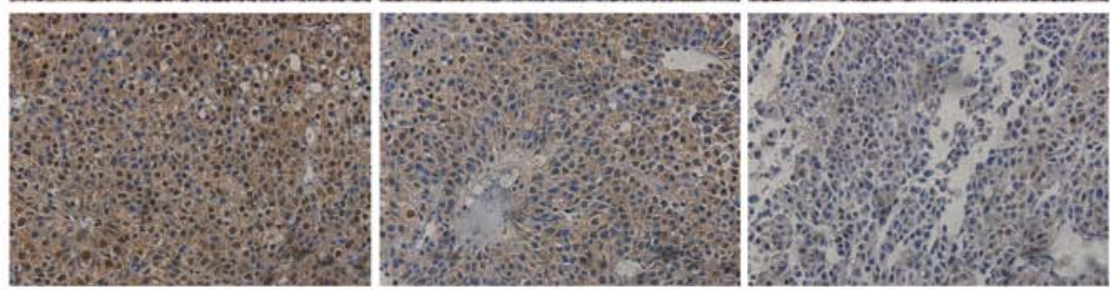

B

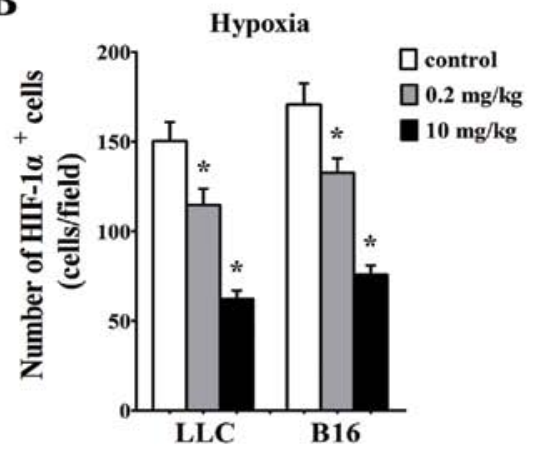

C

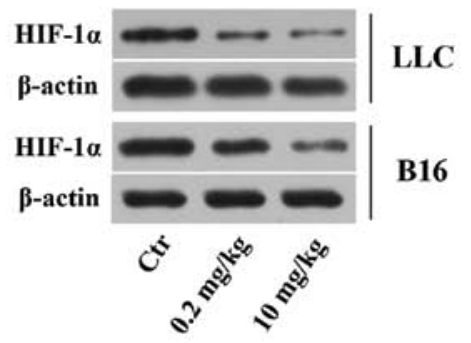

D

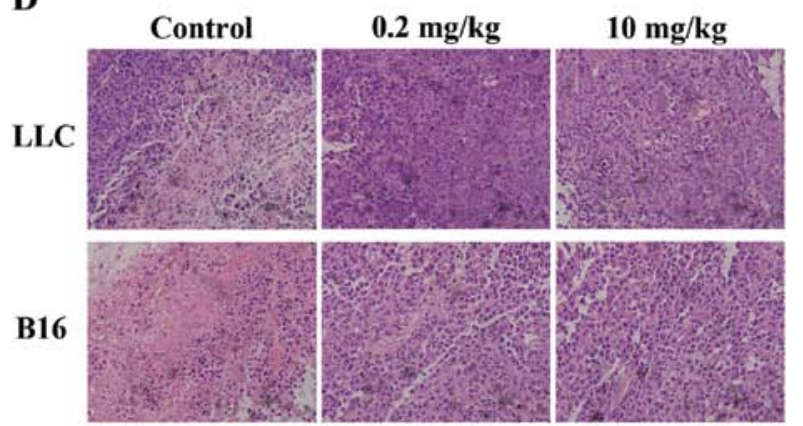

$\mathbf{E}$

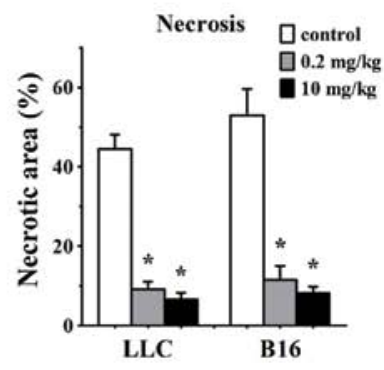

Figure 4. Effect of simvastatin on tumor tissues hypoxia and necrosis. (A) Immunohistochemistry staining for HIF-1 $\alpha$ (brown cell nucleus), revealing less hypoxia in 0.2 and $10 \mathrm{mg} / \mathrm{kg}$ simvastatin treated tumors than controls. (B) Analysis of HIF-1 $\alpha$ positive cells (n=6). (C) Representative western blotting data for HIF-1 $\alpha$ revealing reduced HIF-1 $\alpha$ expression in LLC and B16 tumors tissues treated with 0.2 and $10 \mathrm{mg} / \mathrm{kg}$ simvastatin. (D) $\mathrm{H} \& \mathrm{E}$ staining showing less necrosis in simvastatin treated than control LLC and B16 tumors. (E) Quantification of necrotic area (\% of tumor area) (n=6). All optical micrographs were taken at magnification $\mathrm{x} 400$ for each tumor section. ${ }^{*} \mathrm{p}<0.05$ versus corresponding control animals.

Low-dose simvastatin shows a tendency toward promotion of mature pericyte recruitment by upregulating eNOS expression. Pericytes and ECs are two distinct cell types that constitute a core framework of blood vasculature. Coverage of ECs by pericytes renders vessels a more mature, tight and stable pattern and reduces vessel leakiness (25), a favorable feature of tumor vessel normalization. In this regard, we sought to investigate the effect of simvastatin treatment on pericyte coverage in tumor blood vessels. Double stained for CD31 and mature pericyte marker $\alpha$-SMA revealed a large proportion of naked vessels without $\alpha-\mathrm{SMA}^{+}$cells in untreated LLC and B16 tumors, while only $29.5 \pm 3.7$ and $31.3 \pm 2.7 \%$ of the vessels characterized by discontinuous and incomplete perivascular cell coverage were observed in $10 \mathrm{mg} / \mathrm{kg}$ simvastatin-treated LLC and B16 tumors, respectively. Encouragingly, after the $0.2 \mathrm{mg} / \mathrm{kg}$ simvastatin administration, tumor vessels that encased compactly with $\alpha$-SMA+ pericytes were remarkably increased in both tumor types $\left(71.6 \pm 5.2 \%\right.$ of $\alpha-\mathrm{SMA}^{+}$ vessels in LLC tumors; $82.5 \pm 5.2 \%$ in B16 tumors, $\mathrm{p}<0.001$ vs. controls for both, Fig. 5A-C). Since several mechanisms by which pericytes are recruited have been proposed $(22,26)$, we further explored a probable mechanism by which simvastatin promotes pericyte coverage. A potential mechanism for this effect is that administration of statins with a dosage exerting 
A
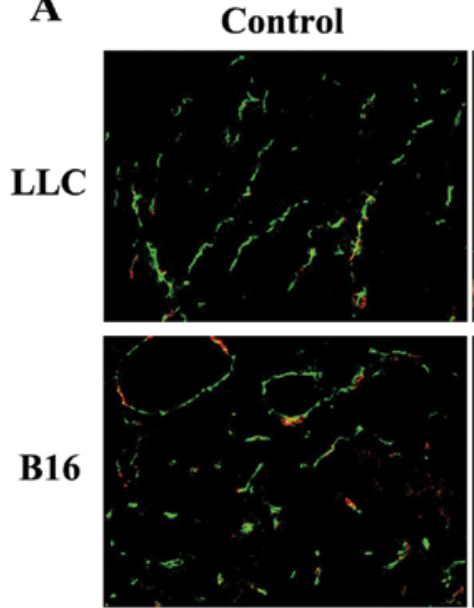

CD31/ $\alpha$-SMA

\section{$0.2 \mathrm{mg} / \mathrm{kg}$}
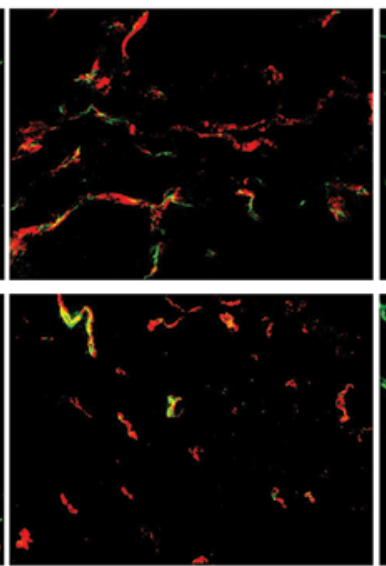

B

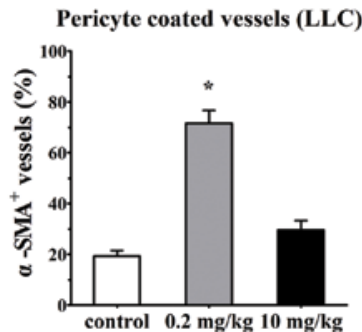

C

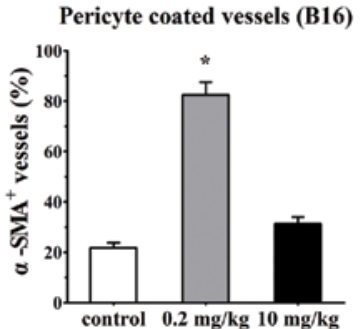

$10 \mathrm{mg} / \mathrm{kg}$
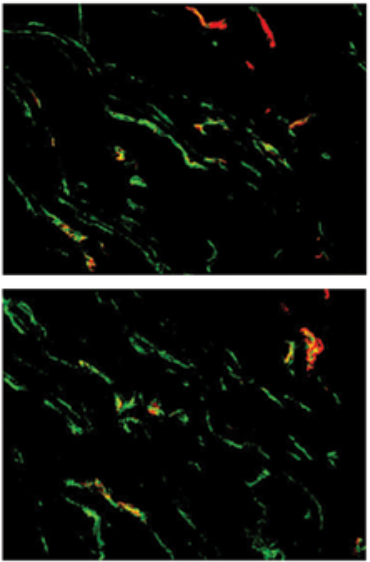

D

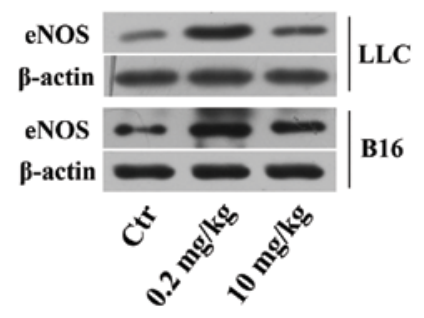

Figure 5. Low-dose simvastatin promotes pericyte recruitment by upregulating eNOS. (A) Double staining for CD31 (green) and $\alpha$-SMA (red) showing significantly more pericyte-covered tumor vessels in $0.2 \mathrm{mg} / \mathrm{kg}$ simvastatin treated than control LLC and B16 tumors, quantified in $(\mathrm{B})$ and $(\mathrm{C})\left(\alpha-\mathrm{SMA}{ }^{+} \mathrm{CD} 31^{+}\right.$ vessels, \% of CD31 $1^{+}$vessels, $\left.\mathrm{n}=6\right)$. (D) Low-dose $(0.2 \mathrm{mg} / \mathrm{kg} / \mathrm{d})$ simvastatin treatment remarkably increased production of eNOS in both tumor types. Digital fluorescent images were captured at magnification $\mathrm{x} 200$. $^{*} \mathrm{p}<0.001$ versus control animals.

proangiogenic effects (low-dose) induces an overexpression of eNOS (21), the predominant source of NO and has emerged as a pivotal factor in mediating endothelium-pericyte interaction and promoting recruitment of pericytes to tumor vessels (22). Consistent with this line, we have observed a significant upregulation of eNOS protein level 7 days after $0.2 \mathrm{mg} / \mathrm{kg}$ simvastatin treatment in LLC and B16 tumors in comparison with low levels of eNOS expression in both untreated animals and a moderate elevation of eNOS in high-dose treated animals, detected by western blotting (Fig. 5D). These data collectively depicted a tendencious role of low-dose simvastatin in normalizing tumor vessel by promoting pericyte recruitment.

High-dose simvastatin highlights a favorable effect of attenuation of vascular permeability by inhibiting VEGF and counteracting ROS generation. Preclinical data demonstrated that high concentration of statins inhibited ECs proliferation and in vivo angiogenesis via diminishing VEGF synthesis $(20,27)$. We reasoned that the anti-angiogenic activity of high-dose simvastatin would favour its function of tumor vessel normalization. As is shown in Fig. 2, vessel density and leakiness were significantly reduced in $10 \mathrm{mg} / \mathrm{kg}$ groups, concurrently, our western blotting data also showed a remarkable downregulation of VEGF in $10 \mathrm{mg} / \mathrm{kg}$ simvastatin treated animals bearing LLC and B16 tumors versus control and low-concentration groups (Fig. 6A). Considering the inhibitory effect of statins on ROS generation and to further elucidate whether ROS are involved in statin-mediated antiangiogenesis in vivo, in situ ROS production was measured using CM-H2DCFDA staining in tumors treated with distinct concentrations of simvastatin and a well-known strong ROS inhibitor (DPI) and then double-staining assay for CD31 and FITC-lectin was done to assess the alterations of morphology, density and vessel perfusion in DPI-treated tumors. The treatment of $10 \mathrm{mg} / \mathrm{kg}$ simvastatin significantly reduced the ROS level by 62.9 and $64.3 \%$ in LLC and B16 tumors in comparison with control ones, respectively ( $\mathrm{p}<0.05$ for both, Fig. 6B and C) and ROS generation in DPI-treated LLC (34.5\% of control) and B16 tumors (26.4\% of control) was obviously lower than the values in control animals ( $p<0.05$, Fig. 6D and E). Similarly, we observed a greater proportion of lectin-stained vessels possessed sharp and distinct outline in DPI-treated LLC $(82.3 \pm 3.9 \%)$ and B16 tumors $(68.1 \pm 5.3 \%)$ accompanied by a remarkably decreased vessel density $(44.2 \pm 5.1$ vessels/ $\mathrm{mm}^{2}$ in DPI-treated LLC tumors; $38.6 \pm 5.9$ vessels $/ \mathrm{mm}^{2}$ in DPI-treated B16 tumors), as compared with the values in controls ( $p<0.05$ for both comparisons, Fig. 6F-H). Moreover, addition of DPI significantly deceased VEGF and HIF-1 $\alpha$ protein level in both tumors (Fig. 6I). These results confirmed that high-dose simvastatin mediated anti-angiogenic effect and downregulation of VEGF, partly through counteracting ROS in tumor microenvironment, which taken together may 
$\mathbf{A}$

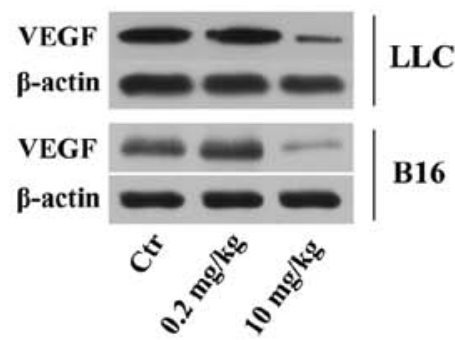

B
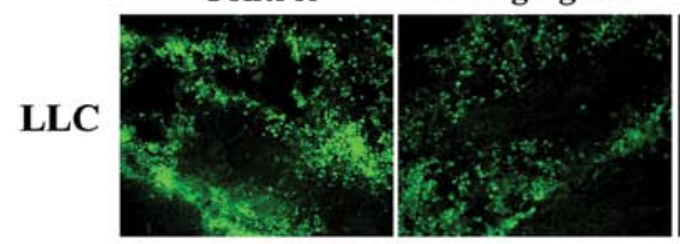

$10 \mathrm{mg} / \mathrm{kg} \mathrm{Sim}$

B16

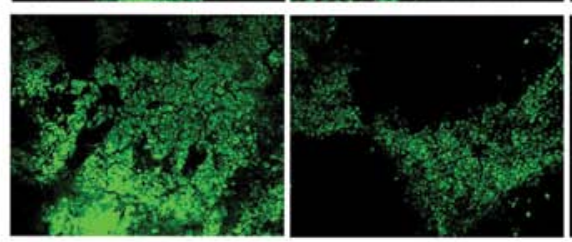

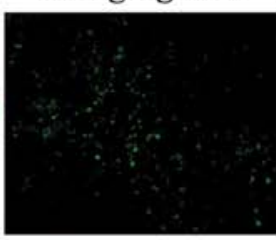

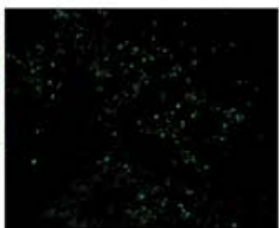

C

Fluorescent intensity of DCF

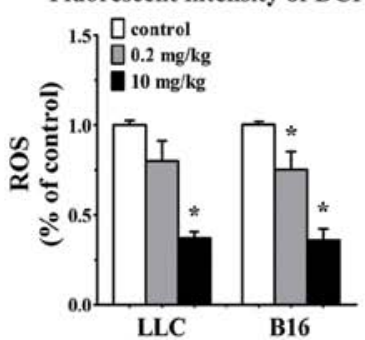

D

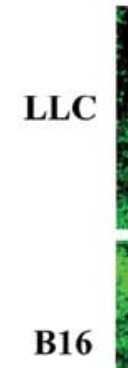

Control
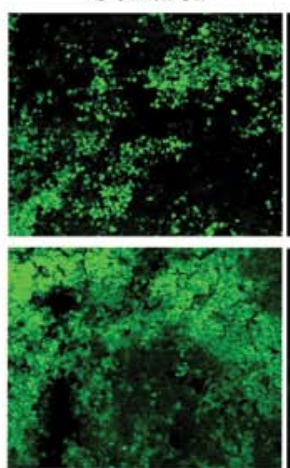

DPI

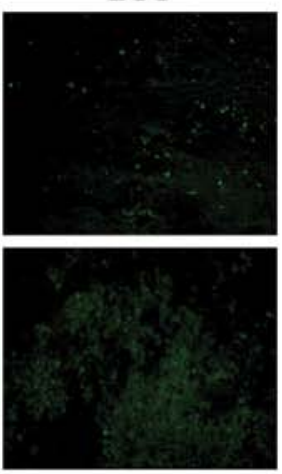

$\mathbf{E}$

Fluorescent intensity of DCF

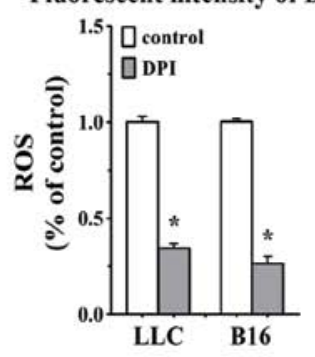

$\mathbf{F}$

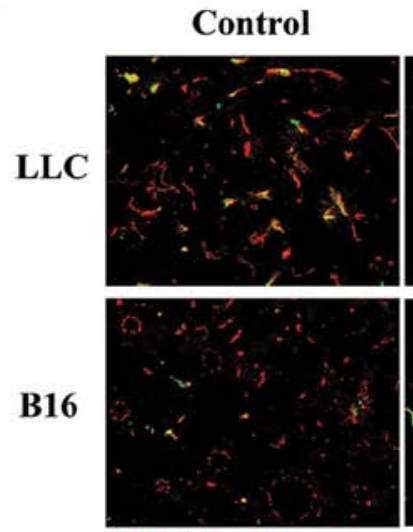

CD31/Lectin
DPI
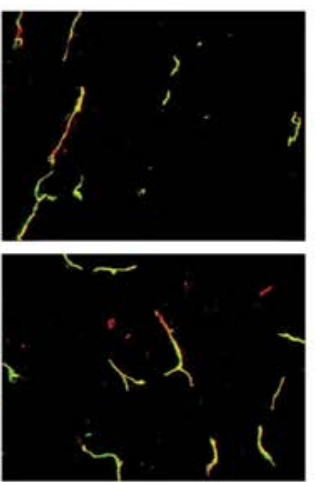

G

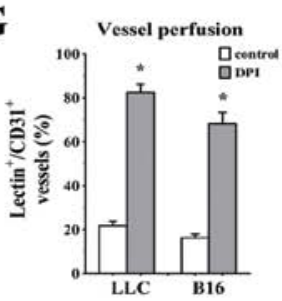

I

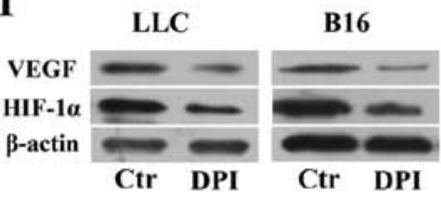

Figure 6. High-dose simvastatin downregulates VEGF via counteracting ROS and improves tumor vessel perfusion. (A) Reduced production of VEGF in high-dose simvastatin (10 mg/kg/d) treated tumors. (B and D) Representative images of DCF (green) indicating in situ ROS generation in simvastatin (B) and DPI (D) treated, freshly prepared tumor sections, revealing dramatically decreased ROS production in $10 \mathrm{mg} / \mathrm{kg}$ simvastatin and DPI treated tumors. (C and E) Semi-quantification of fluorescent intensity of DCF (n=8). (F) Staining for CD31 (red) and FITC-labeled lectin (green) showing more perfused vessels in DPI-treated tumors, quantified in $(\mathrm{G})\left(\%\right.$ of $\mathrm{CD} 31^{+}$vessels, $\left.\mathrm{n}=8\right)$. Significantly decreased vessel density $(\mathrm{H}, \mathrm{n}=8)$ and downregulation of VEGF and HIF-1 $\alpha$ in DPI-treated LLC and B16 tumors (I). Fluorescent images were magnified x 200. *p $<0.05$ versus controls.

explain the ameliorating efficacy of high-dose simvastatin on tumor vessel hyperpermeability.

Greater effect of cytotoxic chemotherapeutics on tumors when given together with simvastatin. A predicted consequence of increased blood perfusion to tumor would be enhanced drug delivery, accordingly, we decided to examine if this change of vessel function following simvastatin treatment has obvious clinical implications for the combination therapy of simv- astatin and chemotherapeutics. Mice bearing LLC and B16 tumors were treated with cisplatin and CTX one day after simvastatin administration. All animals were sacrificed at the end of treatment and tumor size was then assessed. LLC tumors treated with combined modality grew slower than those treated with single chemotherapeutics as the tumor volumes in control and cisplatin-only treatment groups were $742.94 \pm 21.9 \mathrm{~mm}^{3}$ and $480.64 \pm 26.9 \mathrm{~mm}^{3}$ at the end of treatment, respectively. Administration of $0.2 \mathrm{mg} / \mathrm{kg}$ simvastatin 
$\mathbf{A}$

\section{LLC tumor volume}

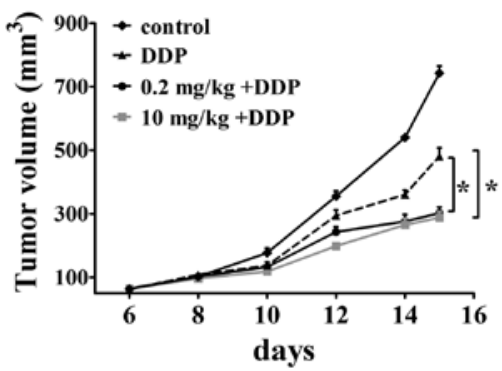

B

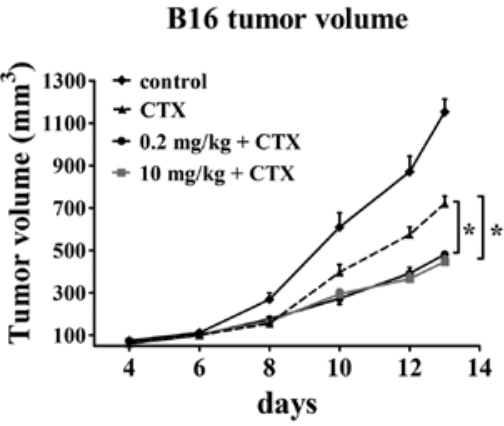

Figure 7. Synergistic effect of simvastatin and chemotherapeutics on tumor growth inhibition. Chemotherapeutics combined with 0.2 or $10 \mathrm{mg} / \mathrm{kg}$ simvastatin shows slower growth of LLC (A) and B16 tumors (B) than chemotherapeutics alone $(\mathrm{n}=10)$. $\mathrm{p}<0.05$ versus corresponding single chemotherapeutic groups.

followed by cisplatin was effective in reducing the tumor volume $\left(302.03 \pm 19.2 \mathrm{~mm}^{3}\right)$. Addition of cisplatin to $10 \mathrm{mg} /$ $\mathrm{kg}$ simvastatin groups showed maximum inhibition of tumor growth $\left(288.27 \pm 18.1 \mathrm{~mm}^{3}\right)$ by 61 and $40 \%$ in comparison with control and single cisplatin-treated groups $(\mathrm{p}<0.05$, Fig. 7A). An increased susceptibility to cytotoxic agent was also observed in B16 tumors that are sensitive to CTX. Single CTX treatment had only a modest effect on tumor growth inhibition (final tumor volume $720.9 \pm 35.5 \mathrm{~mm}^{3}$ vs. $1153.4 \pm 60.5 \mathrm{~mm}^{3}$ in control groups). CTX treatment significantly decreased tumor growth by $58 \%$ (final tumor volume $481.7 \pm 15.6 \mathrm{~mm}^{3}$ ) when given $24 \mathrm{~h}$ after $0.2 \mathrm{mg} / \mathrm{kg}$ simvastatin treatment as compared with control. The synergistic antitumor effect was improved when CTX was administered together with $10 \mathrm{mg} / \mathrm{kg}$ simvastatin (tumor volume $38.7 \%$ of control and $62.6 \%$ of single CTX groups, final volume $446.9 \pm 26 \mathrm{~mm}^{3}, \mathrm{p}<0.05$, Fig. 7B). These findings were consistent with the hypothesis that simvastatinmediated increase of tumor vessel perfusion correlates well with increased efficacy of chemotherapeutic drugs in tumor tissues.

\section{Discussion}

Overall, our data identified a pleiotropic regulatory role for simvastatin on tumor vascular structure and function in a dose-dependent manner, which involves a shift from a hostile pattern of tumor vasculature to a more mature or normalized phenotype and enhanced response to chemotherapeutics. Underlying these activities are distinct potential mechanisms varying with concentrations of simvastatin. On the one hand upregulation of eNOS and derivate NO, stimulated by low-dose simvastatin, promoted pericyte recruitment and the subsequent stabilization of tumor blood vessels, but vessel leakage was remarkably abrogated via normalization of ROS production and inhibiting VEGF in tumor milieu treated by high-dose simvastatin. These data provide insight into the potential use of simvastatin as a vasculoprotective agent.

It is now well established that tumor vessels are immature, mal-shaped and have a disorderly structure with a leaky EC lining that leads to elevated interstitial fluid pressure (IFP) (28). These abnormalities within tumors not only compromise the delivery of therapeutic agents $(29,30)$, but also facilitate metastatic spread (31). Proper incorporation of mural cells into the vessel wall is an indispensable step in the process of tumor vessel normalization. Platelet-derived growth factors (PDGFs), VEGF and angiopoietin 1 (Ang1)/Tie2 are well known cytokines that are involved in pericyte recruitment (32-34). It is noteworthy that $\mathrm{NO}$ has yet been documented to promote vessel maturation by increasing mural cell coverage in B16 melanomas (22). Several reports of both clinical and experimental tumor models have shown that NO plays an important role as a proangiogenic factor in mediating branching and longitudinal extension of blood vessels $(22,35)$, NO mediates endothelialmural cell interaction and induces mural cell recruitment and then a stable functional vessel network is established in tumors. Additionally, Kashiwagi and collaborators have confirmed that vessels of B16 tumors grown in $\mathrm{eNOS}^{-{ }^{--}}$mice have less mural cell coverage with relatively larger vessel diameter compared with vessels in $w t$ B16 tumor-bearing mice, which indicated that eNOS, the predominant source of NO in ECs, is a key modulator involved in above-mentioned effect (22). Here, we have shown that low-dose simvastatin can promote pericyte recruitment with concomitant overexpression of eNOS in the tumor models, despite an increase of vessel density was observed. These actions exactly coincided with the reported regulatory role of eNOS and NO in tumor vessel maturation. Also, it has been reported that low-dose simvastatin $(1 \mathrm{mg} / \mathrm{kg}$, daily) treatment maintained vascular integrity in ischemic brain tissues and significantly reduced blood-brain barrier leakage by increasing Ang1/Tie2 expression, which further supports the role of low-dose simvastatin in vascular stabilization (26). Thus, in view of upregulation of eNOS and protective activities of tumor vessels after lowdose simvastatin treatment, our data are indirectly indicative of the hypothesis that low-dose simvastatin promotes tumor vessel normalization and $\mathrm{NO}$-induced pericyte recruitment is a probable candidate factor.

As a consequence of maturation of vessel structure, efficient tumor blood flow is considered to be an important factor contributing to increased tumor drug delivery. Emerging evidence supports the promotional role of statins on blood flow both in preclinical and clinical experiments. As revealed by Doppler imaging, statins can improve ocular blood flow velocities in patients with DR and preserve blood flow in ischemic limbs $(36,37)$. In addition, augmentation of blood perfusion was reported in subcutaneously inoculated colon cancer models treated with statins (38). Similarly, in our experiments, we found that 7 days after both low- and high-dose simvastatin treatments, tumor blood flow or vessel 
perfusion were markedly improved compared with controls. Given that eNOS-derived NO can serve in its well known capacity as a vasodilator to reduce vascular resistance and potently increase microvascular blood volume and flow (39), as well as the pericytes with abnormal phenotypes could impair tumor blood flow (40), the improvement of tumor blood flow after low-dose simvastatin treatment accompanied by upregulation of eNOS is comprehensible. Moreover, eNOS-derived $\mathrm{NO}$ functions as a second messenger in VEGF signaling and is necessary for the activities of VEGF, such as stimulation of angiogenesis, which is in part, responsible for the increased tumor blood flow (41). Consequently, these data gave rise to a speculative conclusion that the concept of vascular normalization is specialized in the case of low dose simvastatin treatment and conceived as increased neovessel branches, however, most of which are concomitantly covered by pericytes and abundant of blood perfusion.

Impairment of equilibrium of various proangiogenic and anti-angiogenic factors in tumors mostly contributes to relentless development of aberrant vessels. Judicious modification of this imbalance may normalize tumor vasculature (42). One validated modality to correct these vessel dysfunctions is blockade of VEGF signaling. VEGF, a well known mediator that promotes proliferation of ECs, contributes to excessive tumor vessel permeability. Conversely, anti-VEGF therapy can reduce tumor vessel leakage, resulting in a drop in intratumoral IFP and in turn improve oxygenation and drug penetration in tumors $(43,44)$, providing a rationale for clinical use of VEGF-targeted agents in terms of vascular normalization in patients with cancer and/or other pathological angiogenic diseases, exemplified as DR $(45,46)$. Accumulating reports clearly showed that bevacizumab, administered by intraocular injection, has a global effect in normalizing the pathologic intraocular environment and changing the immature, fenestrated vessels toward a normalized status by neutralizing VEGF in ocular disease $(45,47)$. Coincidentally, as equally possessed with antagonistic action against VEGF, high-dose statins have been widely used in treating DR and complications both in clinical settings and experimental models, in consideration of its activities in decreasing retinal neovascularization, retaining endothelial barrier integrity and eventually reducing the area of hypoxia $(13,48)$. Thus, it is reasonable to infer that the beneficial effects of high-dose simvastatin are also applicable to neovessels of tumor. This hypothesis is in line with our present work that administration of a high-dose simvastatin to tumor-bearing mice resulted in significant decrease in the number of tumor vessels by a mechanism involving abrogation of VEGF. During the simvastatin-induced profound vascular regression, the remaining vessels tend to have a more normal structure with dramatically decreased permeability and it resembled the response to bevacizumab by destroying immature vessels in case of pathological ocular and tumor neoangiogenesis. The above might serve to explain the improvement of blood perfusion or flow in high-dose simvastatin treated tumor models in our study. Uniquely, however, as an anti-oxidant, highdose simvastatin is also competent in counteracting oxygen radicals. It was reported that spontaneous overproduction of ROS in tumor cells was required for inducing angiogenesis and tumor growth through the expression of VEGF and
HIF-1 $\alpha$ and this cascade effect was reversible when ROS was removed by specific inhibitors, such as DPI (19). Consistent with this, we found that B16 and LLC tumor tissues have much higher generation of ROS and that high-dose simvastatin or DPI treatment led to a significant decrease of VEGF and HIF-1 $\alpha$ expression when ROS was diminished. More functional tumor vessels with decreased vessel number were presented in DPI-treated animals. In addition, elevated ROS was shown to trigger ECs apoptosis that resulted in the loss of vascular barrier integrity. Application of anti-oxidants may abrogate the ROS-induced impairment of endothelial barrier $(16,49)$. These data are intriguing and together implicate the critical role of endogenous ROS in abnormalities of tumor vessel structure and functions. The anti-angiogenic efficacy of high-dose simvastatin as well as its underlying mechanism by scavenging redundant ROS might be a novel potential way to regulate vessel stabilization and normal vascular barrier function.

In response to improved intratumoral perfusion, there was a relative lack of hypoxia and necrosis within sections of simvastatin-treated tumors tissues and these alterations may indeed lead to a beneficial clinical implication of enhanced efficacy of systemic chemotherapy, as indicated in our results, simvastatin has an additive or synergistic effect in cancer therapy when used in combination with traditional chemotherapeutics. Statins are commonly-used drugs for treating and/or preventing cardiovascular diseases due to its cholesterol-lowing effect. However, increasing evidence suggests that lipid-independent and pleiotropic activities of statins in anti-angiogenesis, cytostasis and repression of tumor metastases may facilitate its anticancer properties in a variety of tumor types $(50,51)$. In the present study, we have demonstrated that simvastatin with both low and high dosages can mediate remodeling and stabilization of tumor vessels, although through differentially ascendant molecular basis and distinct patterns of manifestation.

Our data strongly support the role of statins, like simvastatin, as a promising therapeutic option not only for retina neoangiogenesis but also for use in monotherapy or combination therapy for the treatment of solid tumors. Given the limitations that the favorable pattens of vessel phenotype beyond low and high-dose simvastatin treatments are just some parts of the typical normalized presentations, as assessed from distinct angles respectively and it is not yet clear which medication modality contributes more significantly to tumor vessel normalization, further investigations should be focused on ascertaining the appropriate simvastatin dosing regimen that is suitable for clinical application and optimizing the duration and schedule of the therapy.

\section{Acknowledgements}

We are thankful to Dr Kai Hong (Department of Medical Ultrasound, Tongji Hospital, Tongji Medical College of Huazhong University of Science and Technology) for his excellent technical assistance and would also like to acknowledge the Department of Experimental Zoology, Tongji Medical College, for their helpful assistance with animal maintenance. This study was supported by grants from the National Nature Science Foundation of China (no. 30973473). 


\section{References}

1. Carmeliet P and Jain RK: Principles and mechanisms of vessel normalization for cancer and other angiogenic diseases. Nat Rev Drug Discov 10: 417-427, 2011.

2. Crawford TN, Alfaro DV III, Kerrison JB and Jablon EP: Diabetic retinopathy and angiogenesis. Curr Diabetes Rev 5: 8-13, 2009.

3. Fukumura D, Duda DG, Munn LL and Jain RK: Tumor microvasculature and microenvironment: novel insights through intravital imaging in pre-clinical models. Microcirculation 17: 206-225, 2010

4. Hamzah J, Jugold M, Kiessling F, Rigby P, Manzur M, Marti HH, Rabie T, Kaden S, Gröne HJ, Hämmerling GJ, Arnold B and Ganss R: Vascular normalization in Rgs5-deficient tumors promotes immune destruction. Nature 453: 410-414, 2008.

5. De La Cruz JP, Gonzalez-Correa JA, Guerrero A and de la Cuesta FS: Pharmacological approach to diabetic retinopathy. Diabetes Metab Res Rev 20: 91-113, 2004.

6. Feng Y, vom Hagen F, Pfister F, Djokic S, Hoffmann S, Back W, Wagner P, Lin J, Deutsch U and Hammes HP: Impaired pericyte recruitment and abnormal retinal angiogenesis as a result of angiopoietin-2 overexpression. Thromb Haemost 97: 99-108, 2007.

7. Durham JT and Herman IM: Microvascular modifications in diabetic retinopathy. Curr Diab Rep 11: 253-264, 2011.

8. Jain RK: Normalizing tumor vasculature with anti-angiogenic therapy: a new paradigm for combination therapy. Nat Med 7 : 987-989, 2001

9. Mao Y, Kiss S, Boyer JL, Hackett NR, Qiu J, Carbone A, Mezey JG, Kaminsky SM, D'Amico DJ and Crystal RG: Persistent suppression of ocular neovascularization with intravitreal administration of AAVrh.10 coding for bevacizumab Hum Gene Ther 22: 1525-1235, 2011.

10. Zheng Z, Chen H, Wang H, Ke B, Zheng B, Li Q, Li P, Su L, Gu Q and Xu X: Improvement of retinal vascular injury in diabetic rats by statins is associated with the inhibition of mitochondrial reactive oxygen species pathway mediated by peroxisome proliferator-activated receptor $\gamma$ coactivator $1 \alpha$. Diabetes 59: 2315-2325, 2010

11. Li J, Wang JJ, Chen D, Mott R, Yu Q, Ma JX and Zhang SX: Systemic administration of HMG-CoA reductase inhibitor protects the blood-retinal barrier and ameliorates retinal inflammation in type 2 diabetes. Exp Eye Res 89: 71-78, 2009.

12. Wong WW, Dimitroulakos J, minden MD and Penn LZ: HMG-CoA reductase inhibitors and the malignant cell: the statin family of drugs as triggers of tumor-specific apoptosis. Leukemia 16: 508-519, 2002.

13. Li J, Wang JJ, Yu Q, Chen K, Mahadev K and Zhang SX: Inhibition of reactive oxygen species by lovastatin downregulates vascular endothelial growth factor expression and ameliorates blood-retinal barrier breakdown in $\mathrm{db} / \mathrm{db}$ mice role of NADPH oxidase 4. Diabetes 59: 1528-1538, 2010.

14. Oliveira VN, Bessa A, Jorge ML, Oliveira RJ, de Mello MT, De Agostini GG, Jorge PT and Espindola FS: The effect of different training programs on antioxidant status, oxidative stress and metabolic control in type 2 diabetes. Appl Physiol Nutr Metab 37: 334-344, 2012.

15. Woo DK, Green PD, Santos JH, D'Souza AD, Walther Z, Martin WD, Christian BE, Chandel NS and Shadel GS: Mitochondrial genome instability and ROS enhance intestinal tumorigenesis in APC(Min/+) mice. Am J Pathol 180: 24-31, 2012.

16. Lin RZ, Wang TP, Hung RJ, Chuang YJ, Chien CC and Chang HY: Tumor-induced endothelial cell apoptosis: roles of NAD(P)H oxidase-derived reactive oxygen species. J Cell Physiol 226: 1750-1762, 2011.

17. Deng B, Xie S, Wang J, Xia Z and Nie R: Inhibition of protein kinase $C \beta 2$ prevents tumor necrosis factor- $\alpha$-induced apoptosis and oxidative stress in endothelial cells: The role of NADPH oxidase subunits. J Vasc Res 49: 144-159, 2012.

18. Kojima H, Otani A, Oishi A, Makiyama Y, Nakagawa S and Yoshimura N: Granulocyte colony-stimulating factor attenuates oxidative stress-induced apoptosis in vascular endothelial cells and exhibits functional and morphologic protective effect in oxygen-induced retinopathy. Blood 117: 1091-1100, 2011.

19. Xia C, Meng Q, Liu LZ, Rojanasakul Y, Wang XR and Jiang BH: Reactive oxygen species regulate angiogenesis and tumor growth through vascular endothelial growth factor. Cancer Res 67: 10823-10830, 2007.
20. Weis M, Heeschen C, Glassford AJ and Cooke JP: Statins have biphasic effects on angiogenesis. Circulation 105: 739-745, 2002.

21. Meda C, Plank C, Mykhaylyk O, Schmidt K and Mayer B: Effects of statins on nitric oxide/cGMP signaling in human umbilical vein endothelial cells. Pharmacol Rep 62: 100-112, 2010.

22. Kashiwagi S, Izumi Y, Gohongi T, Demou ZN, Xu L, Huang PL, Buerk DG, Munn LL, Jain RK and Fukumura D: NO mediates mural cell recruitment and vessel morphogenesis in murine melanomas and tissue-engineered blood vessels. J Clin Invest 115: 1816-1827, 2005.

23. Sadeghi MM, Collinge M, Pardi R and Bender JR: Simvastatin modulates cytokine-mediated endothelial cell adhesion molecule induction: involvement of an inhibitory $\mathrm{G}$ protein. $\mathrm{J}$ Immunol 165: 2712-2718, 2000.

24. Mills EM, Takeda K, Yu ZX, Ferrans V, Katagiri Y, Jiang H, Lavigne MC, Leto TL and Guroff G: Nerve growth factor treatment prevents the increase in superoxide produced by epidermal growth factor in PC12 cells. J Biol Chem 273 22165-22168, 1998.

25. Gerhardt $\mathrm{H}$ and Semb H: Pericytes: gatekeepers in tumour cell metastasis? J Mol Med 86: 135-144, 2008.

26. Chen J, Cui X, Zacharek A and Chopp M: Increasing Ang1/Tie2 expression by simvastatin treatment induces vascular stabilization and neuroblast migration after stroke. J Cell Mol Med 13: 1348-1357, 2009

27. Vincent L, Chen W, Hong L, Mirshahi F, Mishal Z, MirshahiKhorassani T, Vannier JP, Soria J and Soria C: Inhibition of endothelial cell migration by cerivastatin, an HMG-CoA reductase inhibitor: contribution to its anti-angiogenic effect. FEBS Lett 495: 159-166, 2001

28. De Bock K, Cauwenberghs S and Carmeliet P: Vessel abnormalization: another hallmark of cancer? Molecular mechanisms and therapeutic implications. Curr Opin Genet Dev 21: 73-79, 2010.

29. Tong RT, Boucher Y, Kozin SV, Winkler F, Hicklin DJ and Jain RK: Vascular normalization by vascular endothelial growth factor receptor 2 blockade induces a pressure gradient across the vasculature and improves drug penetration in tumors. Cancer Res 64: 3731-3736, 2004.

30. Rapisarda A and Melillo G: Role of the hypoxic tumor microenvironment in the resistance to anti-angiogenic therapies. Drug Resist Updat 12: 74-80, 2009

31. Pennacchietti S, Michieli P, Galluzzo M, Mazzone M, Giordano S and Comoglio PM: Hypoxia promotes invasive growth by transcriptional activation of the met protooncogene. Cancer Cell 3 : 347-361, 2003

32. Raza A, Franklin MJ and Dudek AZ: Pericytes and vessel maturation during tumor angiogenesis and metastasis. Am J Hematol 85: 593-598, 2010

33. Greenberg JI and Cheresh DA: VEGF as an inhibitor of tumor vessel maturation: implications for cancer therapy. Expert Opin Biol Ther 9: 1347-1356, 2009.

34. Winkler F, Kozin SV, Tong RT, Chae SS, Booth MF, Garkavtsev I, Xu L, Hicklin DJ, Fukumura D, di Tomaso E, Munn LL and Jain RK: Kinetics of vascular normalization by VEGFR2 blockade governs brain tumor response to radiation: role of oxygenation, angiopoietin-1 and matrix metalloproteinases. Cancer Cell 6: 553-563, 2004.

35. Jadeski LC and Lala PK: Nitric oxide synthase inhibition by NG-nitro-L-arginine methylester inhibits tumor-induced angiogenesis in mammary tumors. Am J Pathol 155: 1381-1390, 1999.

36. Ozkiris A, Erkiliç K, Koç A and Mistik S: Effect of atorvastatin on ocular blood flow velocities in patients with diabetic retinopathy. Br J Ophthalmol 91: 69-73, 2007.

37. Yu J, deMuinck ED, Zhuang Z, Drinane M, Kauser K, Rubanyi GM, Qian HS, Murata T, Escalante B and Sessa WC: Endothelial nitric oxide synthase is critical for ischemic remodeling, mural cell recruitment and blood flow reserve. Proc Natl Acad Sci USA 102: 10999-11004, 2005.

38. Sata M, Nishimatsu H, Osuga J, Tanaka K, Ishizaka N, Ishibashi S, Hirata Y and Nagai R: Statins augment collateral growth in response to ischemia but they do not promote cancer and atherosclerosis. Hypertension 43: 1214-1220, 2004.

39. Wang N, Ko SH, Chai W, Li G, Barrett EJ, Tao L, Cao W and Liu Z: Resveratrol recruits rat muscle microvasculature via a nitric oxide-dependent mechanism that is blocked by TNF $\alpha$. Am J Physiol Endocrinol Metab 300: E195-E201, 2011.

40. Morikawa S, Baluk P, Kaidoh T, Haskell A, Jain RK and McDonald DM: Abnormalities in pericytes on blood vessels and endothelial sprouts in tumors. Am J Pathol 160: 985-1000, 2002. 
41. Tu YT, Tao J, Liu YQ, Li Y, Huang CZ, Zhang XB and Lin Y: Expression of endothelial nitric oxide synthase and vascular endothelial growth factor in human malignant melanoma and their relation to angiogenesis. Clin Exp Dermatol 31: 413-418, 2006.

42. Jain RK: Normalization of tumor vasculature: an emerging concept in antiangiogenic therapy. Science 307: 58-62, 2005.

43. Dickson PV, Hamner JB, Sims TL, Fraga CH, Ng CY, Rajasekeran S, Hagedorn NL, McCarville MB, Stewart CF and Davidoff AM: Bevacizumab-induced transient remodeling of the vasculature in neuroblastoma xenografts results in improved delivery and efficacy of systemically administered chemotherapy. Clin Cancer Res 13: 3942-3950, 2007.

44. Myers AL, Williams RF, Ng CY, Hartwich JE and Davidoff AM: Bevacizumab-induced tumor vessel remodeling in rhabdomyosarcoma xenografts increases the effectiveness of adjuvant ionizing radiation. J Pediatr Surg 45: 1080-1085, 2010.

45. Kohno R, Hata Y, Mochizuki Y, Arita R, Kawahara S, Kita T, Miyazaki M, Hisatomi T, Ikeda Y, Aiello LP and Ishibashi T: Histopathology of neovascular tissue from eyes with proliferative diabetic retinopathy after intravitreal bevacizumab injection. Am J Ophthalmol 150: 223-229, 2010.
46. Sorensen AG, Emblem KE, Polaskova P, Jennings D, Kim H, Ancukiewicz M, Wang M, Wen PY, Ivy P, Batchelor TT and Jain RK: Increased survival of glioblastoma patients who respond to antiangiogenic therapy with elevated blood perfusion. Cancer Res 72: 402-407, 2012.

47. Sharma RK, Rogojina AT and Chalam KV: Bevacizumab therapy normalizes the pathological intraocular environment beyond neutralizing VEGF. Mol Vis 16: 2175-2184, 2010.

48. Gupta A, Gupta V, Thapar S and Bhansali A: Lipid-lowering drug atorvastatin as an adjunct in the management of diabetic macular edema. Am J Ophthalmol 137: 675-682, 2004.

49. ten Kate M, van der Wal JB, Sluiter W, Hofland LJ, Jeekel J, Sonneveld $\mathrm{P}$ and van Eijck $\mathrm{CH}$ : The role of superoxide anions in the development of distant tumour recurrence. Br J Cancer 95: 1497-1503, 2006.

50. Wang C, Tao W, Wang Y, Bikow J, Lu B, Keating A, Verma S, Parker TG, Han R and Wen XY: Rosuvastatin, identified from a zebrafish chemical genetic screen for antiangiogenic compounds, suppresses the growth of prostate cancer. Eur Urol 58: 418-426, 2010.

51. Mandal CC, Ghosh-Choudhury N, Yoneda T, Choudhury GG and Ghosh-Choudhury N: Simvastatin prevents skeletal metastasis of breast cancer by an antagonistic interplay between p53 and CD44. J Biol Chem 286: 11314-11327, 2011. 